\title{
Electronic Tool Interferences with Electrophysiology for the Psychiatric Disorders Monitoring
}

\author{
Florin Babarada ${ }^{1}$, Cristian Ravariu ${ }^{1}$, Dan Prelipceanu ${ }^{2}$, Bogdan Patrichi ${ }^{2}$, Daniela Manuc ${ }^{3}$, \\ Aurora Salageanu ${ }^{4}$, Iuliana Caras ${ }^{4}$ \\ ${ }^{1}$ Department of Electronics, Polytechnic University of Bucharest, ERG-BioNEC Group, Bucharest, Romania \\ ${ }^{2}$ Department of Psychiatry, Carol Davila University of Medicine and Pharmacy, Bucharest, Romania \\ ${ }^{3}$ Public Health, Carol Davila University of Medicine and Pharmacy, Bucharest, Romania \\ ${ }^{4}$ Cantacuzino National Research and Development Institute for Microbiology and Immunology, Bucharest, Romania
}

\section{Email address:}

babflorin@yahoo.com (F. Babarada), cristian.ravariu@upb.ro (C. Ravariu)

\section{To cite this article:}

Florin Babarada, Cristian Ravariu, Dan Prelipceanu, Bogdan Patrichi, Daniela Manuc, Aurora Salageanu, Iuliana Caras. Electronic Tool Interferences with Electrophysiology for the Psychiatric Disorders Monitoring. American Journal of Bioscience and Bioengineering. Special Issue: Bio-electronics: Biosensors, Biomedical Signal Processing, and Organic Engineering. Vol. 3, No. 3-1, 2015, pp. 14-21.

doi: 10.11648/j.bio.s.2015030301.13

\begin{abstract}
The paper presents a hardware solution of the in vivo electrophysiological signals acquiring and processing, using a continuous data acquisition on PC. The originality of the paper comes from architecture proposal, with some new blocks, which selective amplify and filter biosignals. One of the major problems in the electrophysiological signals monitoring is the impossibility to record the weak signals from deep organs that are covered by noise and by strong cardiac or muscular artefact signals. The analogical processing block is based on a dynamic range compressor, containing the automatic gain control block, so that the high power signals are less amplified than the low components. The following block is a clipper since to capture all the transitions that escape from the dynamic range compressor. At clipper output a low-pass filter is connected since to abruptly cut the high specific bio-frequencies. The data vector recording is performing by strong internal resources microcontroller including ten bits $\mathrm{A} / \mathrm{D}$ conversion port. Through some specific measurements and calibration the chain can be used to capture and then interprets the neuronal signal with well applications in public health monitoring like psychiatric disorders.
\end{abstract}

Keywords: Healthcare, Compressor Technique, Bioinformatics, Signal Processing, Public Heath, Psychiatric

\section{Introduction}

The common techniques from the human electrophysiology are non-invasive, with electrodes placed on the tissue (e.g. metallic electrodes in contact with gastric mucosa in electrogastrography [1] or at cutaneous level in the classical electrocardiography ECG [2]).

Therefore, a main problem arises when the electrodes are placed onto skin: the useful weak signals are buried in high level parasitic signals. The non-invasive electrophysiological methods suffer from noise, collected by the surface electrodes. There are many types of noise to be considered:

- Inherent noise in electronics equipment: It is generated by all electronics equipment and can't be eliminated. It is only reduced by high quality components using. It has a frequency range: 0 - several thousand $\mathrm{Hz},[3]$.

- Ambient noise: The cause is the electromagnetic radiation, with possible sources: radio transmission, electrical wires, optical network, [4]. It has a dominant frequency of $50 \mathrm{~Hz}(60 \mathrm{~Hz})$ and amplitude of $1 \div 3 \mathrm{x}$ EMG signal.

- Motion artefact: It has two main sources: electrode/skin interface and electrode/cable, having a frequency range of $0 \div 20 \mathrm{~Hz}$. It is reducible by a proper circuitry and set-up.

- Inherent instability of signal: All electronics equipment's generate noise and the amplitude is somewhat randomized, being in correlation with the discrete nature of the matter. This noise has a frequency range of $0 \div 20 \mathrm{~Hz}$ and cannot be removed.

In this situation, a gastric signal for instance, recorded at skin level, is hundreds times lower than the parasitic signals.

The most accurate solution is the invasive one, straight to the target organ, using microelectrodes, [5]. Unfortunately, the 
majority of organs are inaccessible without a surgical act, which adds two great disadvantages: the health-state in danger and high costs. This paper presents an analogical processing and digital recording system for low power electrophysiological signals, with the possibility to use them in medical applications like ECG, EEG, EMG and other public health monitoring. For low contact electrodes area, the noise introduced by the electrodes begins to be most significant. As the results of modelling of the ensemble source-electrode, it is recommended for large electrodes area that the amplifier must be implemented by a low noise and distortions, transimpedance amplifier stage followed by one low-pass filter. For very low electrophysiological signals it is necessary a differential amplifier because it has a high common mode rejection of parasitic signals characteristic [6].

\section{Interferences with Psychiatry}

The electrophysiological recordings must be associated with novel hardware and software methods of new parameters extraction useful for clinical biometry. Similar quantitative measurements in the field of life sciences envisages biochemical blood tests by novel biosensors [7], neurotransmitter characterization [8] or monitoring [9], medical imagistic processing [10], up to biosignal processing within electrophysiological equipment's, [11-13]. In this scope the term of "Biometry", admitted as a specialty journal title, suggests a large palette of techniques able to perform a metric recording in the biological sciences [14].

For diagnosis and disorder monitoring, some considerable efforts were done to introduce some biometric parameters in medicine: average reflection coefficient (ARC) measured by laser bio-photometry [15], thermo-graphic technique in inflammatory processes [16] and others [17-19]. New models accompanied by new clinical parameters [20] help to a better patient monitoring, like in the diabetes case [21].

In psychiatry, new biometric parameters could be valuable in diagnosis [22]. On the other hand, the electro-physiological methods can be extrapolated from cellular level [23] to clinical applications [24]. For instance, in the research of schizophrenia, the electrophysiological tests indicated some biometric parameters that can be responsible of differential diagnosis among psychotic disorders [25].

In a sequence of time, thousands of ongoing brain processes are captured by electro-encephalography. Therefore, the brain response to a single stimulus is uncertain and usually it is not visible in the EEG trace of a single trial. To collect the brain response to a stimulus, the clinicians must conduct more than 100 trials, to extract the biometric results associated to the random brain activity. After the statistical averaging, the relevant brain waveforms well-known as the ERP (Event-Related brain Potentials) are collected [26].

During a clinical study, the procedure must appeal to a trial of patients with known diseases and must be compared with a trial with healthy subjects. All the persons have to receive the same task (e.g. a specific motion), while their electroencephalography must be simultaneously recorded. If on the EEG trace, the patients with different diseases statistically present different stimuli as human movement compared to healthy controls, some biometric parameters can be established for a differential diagnosis.

Consistent with prior procedure, some authors revealed that some schizophrenia subjects were impaired in their ability to recognize biological motion. Three Event-Related Potential ERP were examined: P1, N1, and the late positive potential LPP [27].The EEG results showed that biological motion did not influence the earliest stage of visual processing (P1). Although schizophrenia subjects showed the same pattern of (N1) results relative to healthy controls, they were impaired at a later stage (LPP), reflecting a dysfunction in the identification of human form in biological versus non-biological motion stimuli. In spite of many promising investigations that studied the usefulness of qualitative EEG in the differential diagnosis between schizophrenia and others psychosis, the results were not always systematically concluding, especially due to non-identical medical condition. No meta-analysis could be conducted due to the lack of reliable quantification in the studies, excluding Oxford CEBM level 3 non-consecutive studies or cohort-study without consistently-applied reference standard or better exploratory or validating cohort-study [25].

Others authors considered the electroencephalography in relation to attention-deficit/hyperactivity disorders (AD/HD) [28]. The qualitative and quantitative EEG studies are investigated in their inter-relationship with different forms of $\mathrm{AD} / \mathrm{HD}$. The conclusions showed that in terms of resting EEG, elevated relative theta rhythm and reduced relative alpha and beta, together with elevated theta/alpha and theta/beta ratios, are most frequently associated with $\mathrm{AD} / \mathrm{HD}$ disorders. Inside subgroups of $\mathrm{AD} / \mathrm{HD}$, the ratios theta/alpha and theta/beta, provides additional biometric parameters for a differential diagnostic.

Further research to date has identified a substantial number of EEG correlates of $\mathrm{AD} / \mathrm{HD}$ which hold promise for improving our understanding of the brain dysfunctions underlying the disorder [29].

\section{Proposal and Simulations of an Analog Electrophysiological Signal Processor}

As in the case of many concepts from engineering, automatic gain control was discovered by natural selection. Automatic gain control (AGC) is an adaptive system found in many electronic devices. The average output signal level is feedback to adjust the gain to an appropriate level for a range of input signal levels. AGC algorithms often use a proportional-integral-differential controller.

Under a sinusoidal signal stimulation having amplitude higher than the flatting threshold at the clipper input, the output signal is available in fig. 1. 


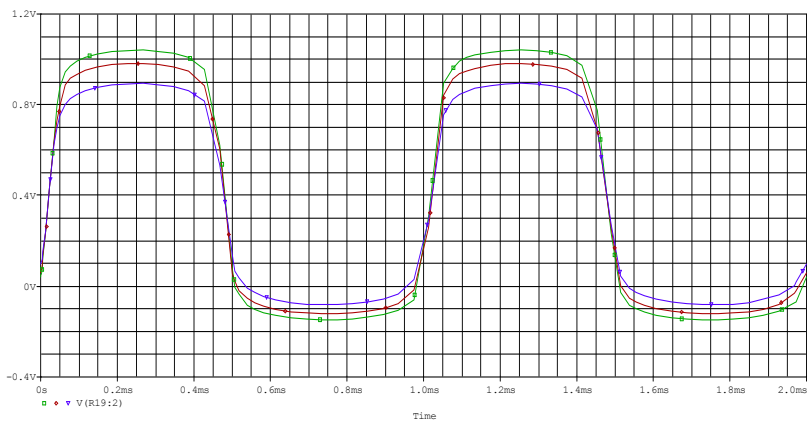

Figure 1. The output signal from the clipper stage.

All three signals are simulated for three different temperatures: $0^{\circ} \mathrm{C}, 20^{\circ} \mathrm{C}$ and $50^{\circ} \mathrm{C}$, revealing a good stability.

Due to the semiconductor devices parameters dependence on temperature, like silicon diodes in forward biasing, the previous green, red and blue curves are quite distinct, in the upper part and bottom part of the output, fig. 1.

For this inconvenient correction, we propose a thermal compensated circuit from fig. 2 . The thermal compensation is ensured by the diode D9 and resistor R22.

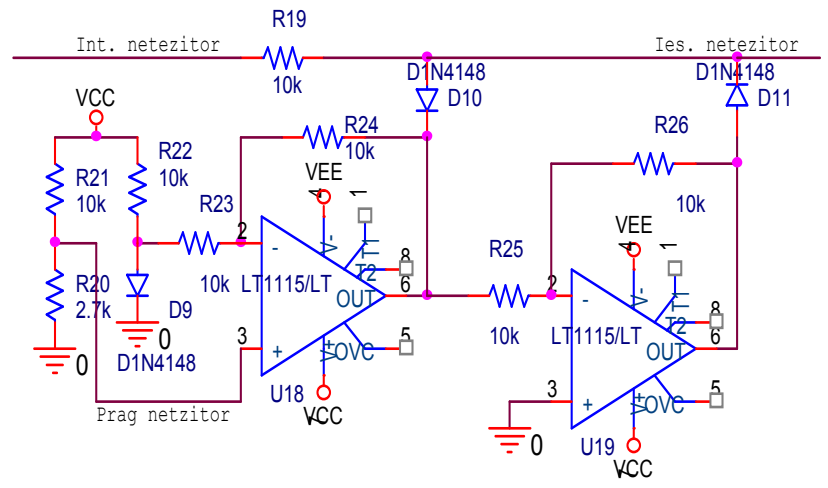

Figure 2. Clipper circuit with thermal compensation.

During a next optimization we interpose a selective amplifier between the command circuit and clipper, fig. 3 .

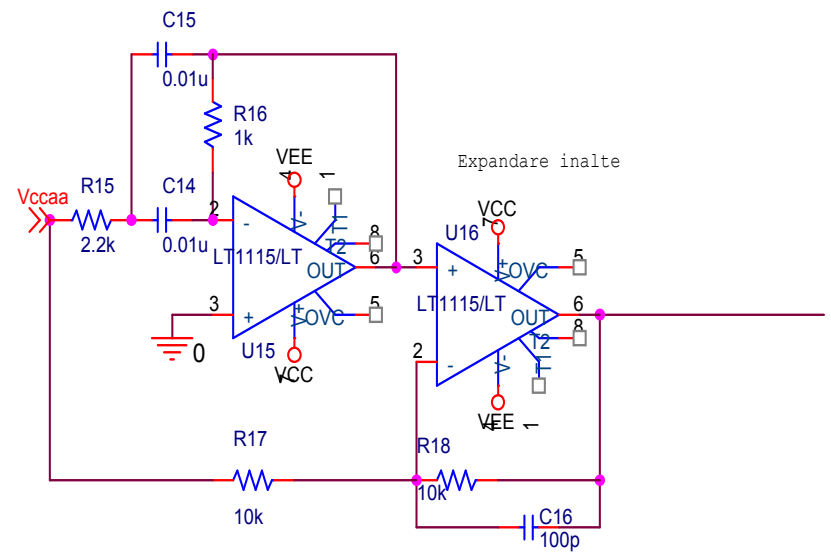

Figure 3. Selective amplifier of higher frequencies.

In this way, another disadvantage has been removed: distortions of the transfer function, produced by the reverse biased diodes at 1-2Volts, are alleviated. When R16 increases, the higher frequencies are emphasized, accordingly with fig. 4.

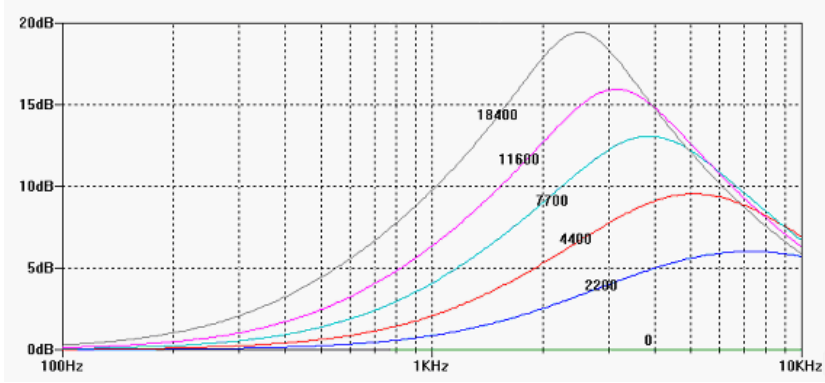

Figure 4. The clipper driver frequency response.

This technique reduces the inter-modulation distortions and allows the high frequencies expansion.

The simulations results that cumulate all previous described benefits are presented in fig. 5 .

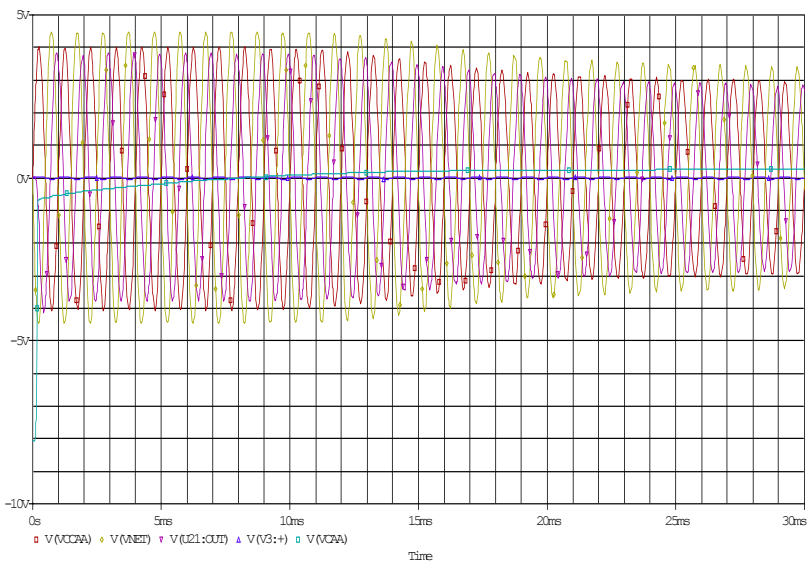

Figure 5. The transient reply; the amplitude of the input signal was $40 \mathrm{mV}$.

The evolution of the command voltage of the compressor, Vcaa can be followed from negative to positive values, besides to the correction moment for the output amplitude.

Frequency response of the entire chain corresponds with the block level simulations and makes the designed behaviour. Thus the compressor frequency response is smooth over $500 \mathrm{KHz}$, the driver stage of clipper emphasizes high frequencies and low-pass filter cut abruptly the frequencies over $3 \mathrm{KHz}$, fig. 6 .

The test of the automatic gain control circuit, contained in the dynamic range compressor, is tested using transient simulations at different input signal amplitudes.

For the input sinusoidal signal amplitude of $10 \mathrm{mV}$, an amplification of the output signal, equal with 100 , is registered. The compression is fulfilled by the stage with $\mathrm{U} 11$ and the $\mathrm{R}_{4}$ and $\mathrm{R}_{5}$ resistances. The command voltage, Vcaa, takes negative values, firstly $-8 \mathrm{~V}$ and after $5 \mathrm{~ms}$ it is changing at $-0.8 \mathrm{~V}$, fig. 7 . 


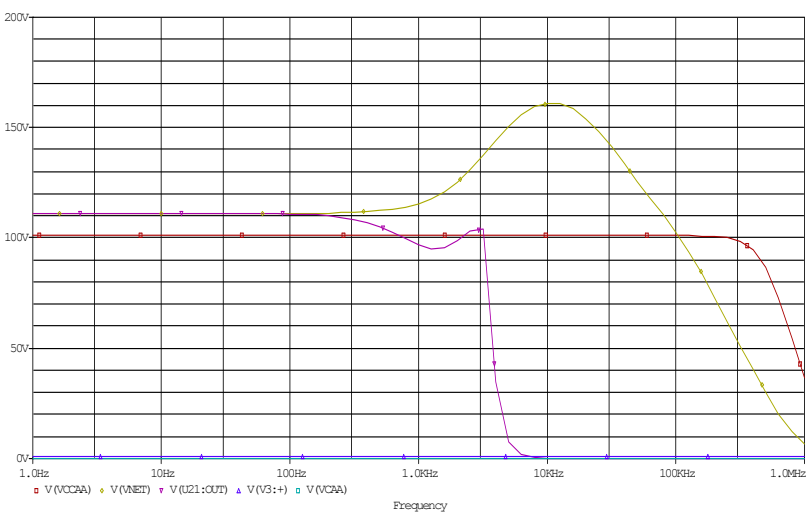

Figure 6. Frequencies response of the whole signal processing chain.

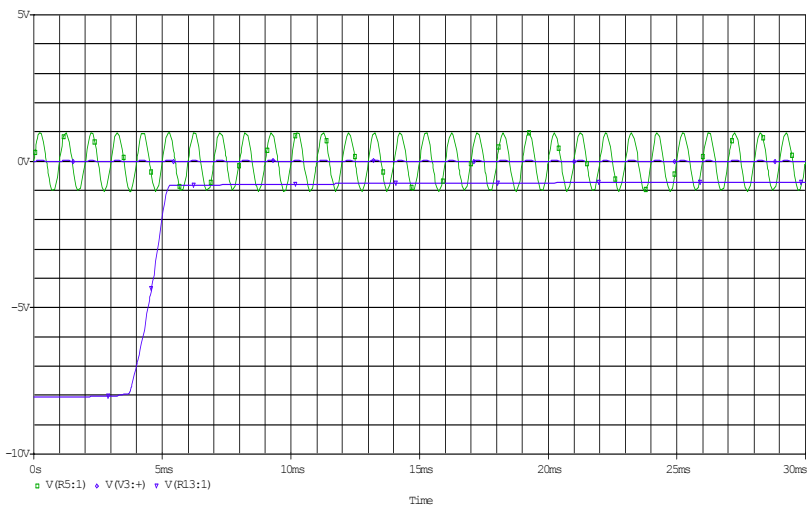

Figure 7. The output voltage Vcaa simulation for an input voltage with the amplitude equal with $10 \mathrm{mV}$.

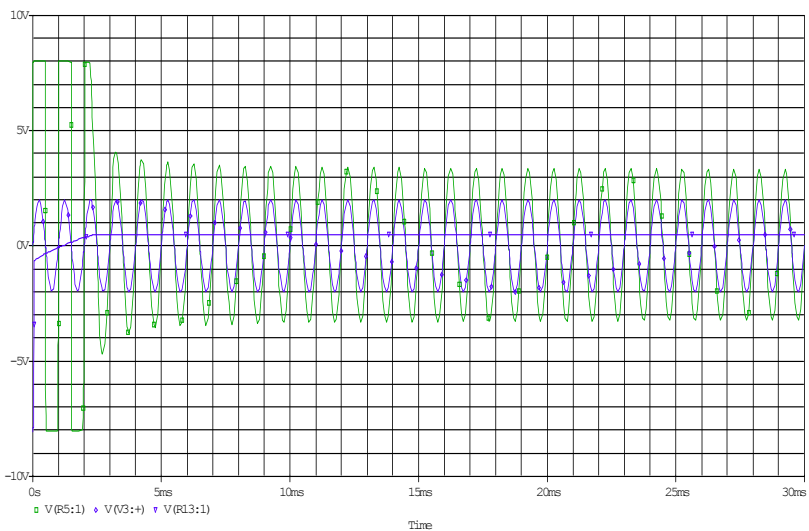

Figure 8. The output voltage Vcaa simulation for an input voltage with the amplitude equal with $2 \mathrm{~V}$.

For the input sinusoidal signal amplitude of $2 \mathrm{~V}$ a new reduction of the amplification of the output signal occurs between $2 \mathrm{~ms}$ up to $4 \mathrm{~ms}$. The Vcaa voltage is negative, approximate of $-8 \mathrm{~V}$ for short time; then increases to a positive value, around $+0.5 \mathrm{~V}$ that reduces the output signal amplitude toward $+3.25 \mathrm{~V}$, fig. 8 .

These simulations prove the dynamic compressor function for the above circuit, which means reduction of the differences between high and low signals levels or amplitudes.

\section{The Digital Interface}

The electrophysiological signals acquiring begins from the source of the bioelectric signals coupled with the electrodes, amplification, processing, analog-digital conversion and data storage in some file format. For the electrophysiological studies, the data storage is necessary, for a long time, as vector data storage.

Acquisition and data storage are performed by an 8-bit microcontroller series AVR (Atmel), namely ATMega32 on a development board that has its own power source, a real time clock circuit, an EEPROM memory, a LED display and a serial interface adapter (RS232 or RS485), fig. 9. This microcontroller has strong internal resources, allowing data acquisition and digital conversion through a 10-bit ADC, provided with eight inputs multiplexer and its own high accuracy reference voltage reference [30].

Different interesting voltages are collected to internal DAC by means of microcontroller port $\mathrm{A}, \mathrm{ADC} 0$ to ADC7. The conversion of analog data to a digital vector is synchronized by an internal clock which allows for choose different sampling rates. A conversion cycle starts by clearing the memory locations for the measured values. After that, every input is converted in a 10 Bits word and temporary stored into the internal RAM memory then the next input is also converted, and so on. At this moment we have an eight 10 Bytes words representing a sample of the analog entry signal. This word is now completed with the conversion time, extracted from the external "Real Time Clock" (RTC), the U10 chip.

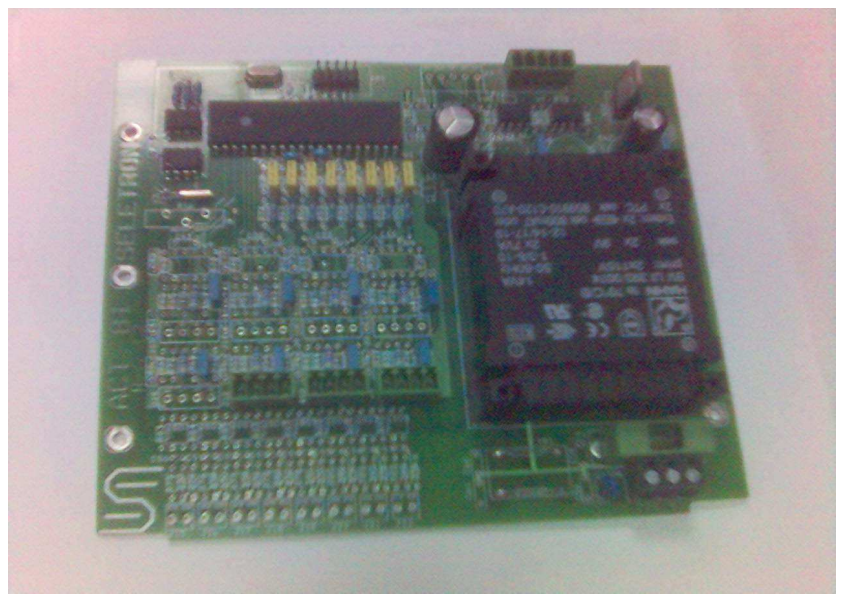

Figure 9. The digital recording module using the microcontroller with integrated port analog-digital converter.

The vector obtained looks like: 5AYYMMDDHHmmsshhV0V1....V7, [31]. The whole record is now 24 bytes long and it is stored into the external flash memory U2. This memory has 65536 bytes allowing for over 2700 records. At a rate of 20 samples/second that means there is enough space for more of 2 minutes of records. The acquired data can be extracted by a serial link, the chip U3 providing for the RS232 specification, including hardware handshake by RTS-CTS pair. The communications parameters 
have been choose to meet the MODBUS specification, as is: 1 START bit, 8 data bits, 9600 Bauds, 1 Even parity bit, 1 STOP bit. During the recording process time information and recorded values are displayed cyclic.

\section{Capabilities of the Global Equipment}

The manufactured electrodes are tested using a multi-channel ADS1298, which work principle was previously depicted, [31], working now on 24-Bit Analog-to-Digital Converter with integrated electro-physiological front-end platform to increase the accuracy.

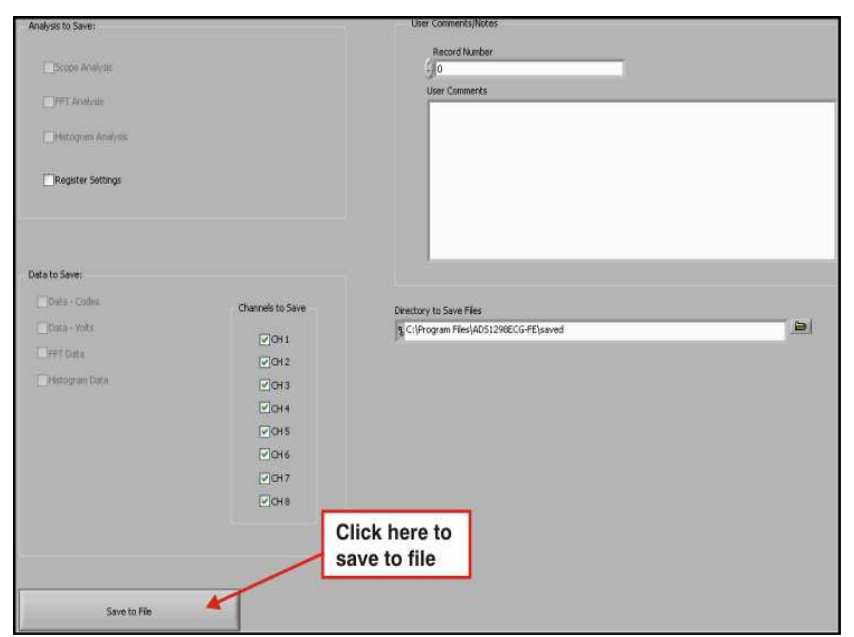

Figure 10. Options for SAVE tab.

The integrate ADS1298 platform is compatible with a dedicated software for general electrophysiology from Texas Instruments, ADS1298ECGFE-PDK Version 1.0.0. This requires the software installation to make available the cropped biosignals storage and representation on a PC monitor using "Save" button, fig. 10. The stored signals can be subsequently processed to extract some biometric parameters useful in differential diagnosis.

The tab GPIO and Other Registers is placed under the analyse tab, including the control from GPIO1 up to GPIO4, for the phase and frequency of respiration, Wilson tracing for amplifier and Goldberger derivation for terminals. The GPIO tab controls four pins of general usage, I/O, fig. 11.

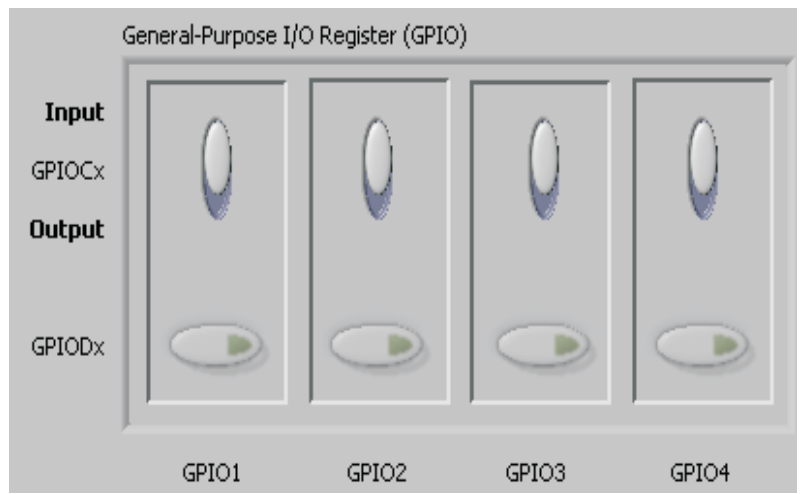

Figure 11. GPIO register inside its window.
If a skin electrode loses the intimate contact so that the series contact resistance abruptly increases, the correspondent bits register records a sub-threshold value in the Status register. This signals the respective electrode separation, changing the colour from green to red, fig. 12.

In figure 13, the Scope mode analysis is revealed for the ECG standard signal for one channel. The Scope mode makes visible the I-III connexions, besides to the derivations Augmented AVF, AVL, AVR, configured after the mode "Normal electrodes".

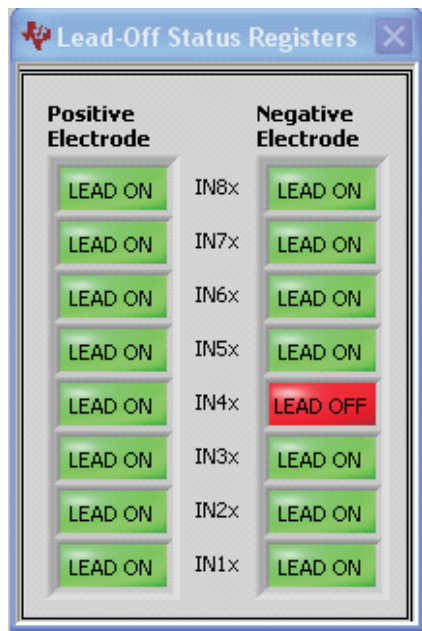

Figure 12. Status register of the electrodes; red colour indicates Lead-Off Status.

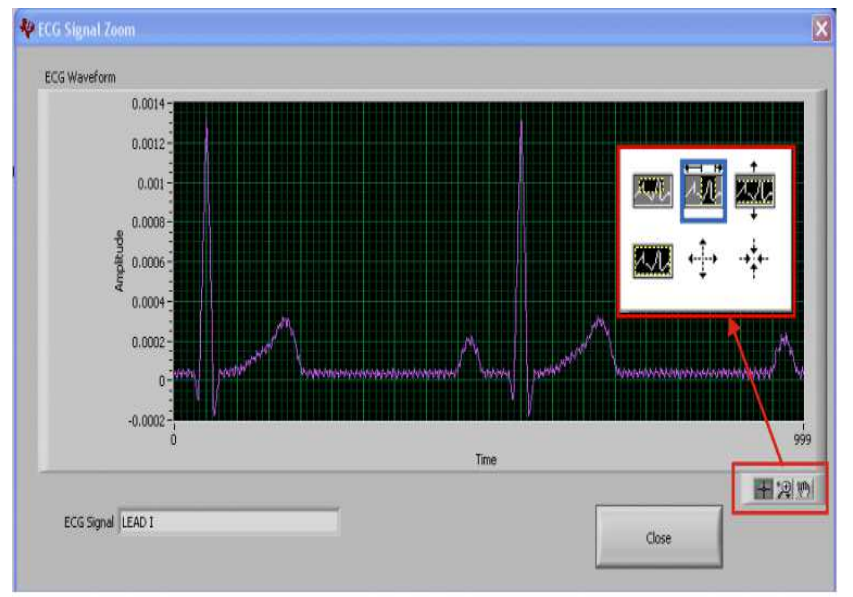

Figure 13. ECG cropped signal in the Scope mode, using one communication channel.

\section{Electrophysiological Vector Recording}

In electrophysiological tests, a special attention is paid to the non-invasive measurements for rapid clinical recordings. Here the biosignal frequency ranges between $0.2 \mathrm{~Hz}$ to $30 \mathrm{~Hz}$. In opposition, accurate measurements, for instance in Electro-Myo-Graphy, is recorded by invasive needles. If a motor unit is touched, the work frequency rises to $0.1 \mathrm{kHz}$, while at cellular level the action potentials variations reaches to $10 \mathrm{kHz},[32]$.

In our tests, the standard ECG electrodes were placed onto 
the abdomen skin, searching the sensitive to others biosignals than ECG. The acquired signals are transmitted to a laptop by a PC interface and displayed, fig. 14.

Between two cardiac beats the signals are more constants. So, the Display mode is suitable, fig. 14, while any additional muscular signals with higher frequency aren't visible. Much more variable signals than an ECG trace become visible using the Scope mode, fig. 15.

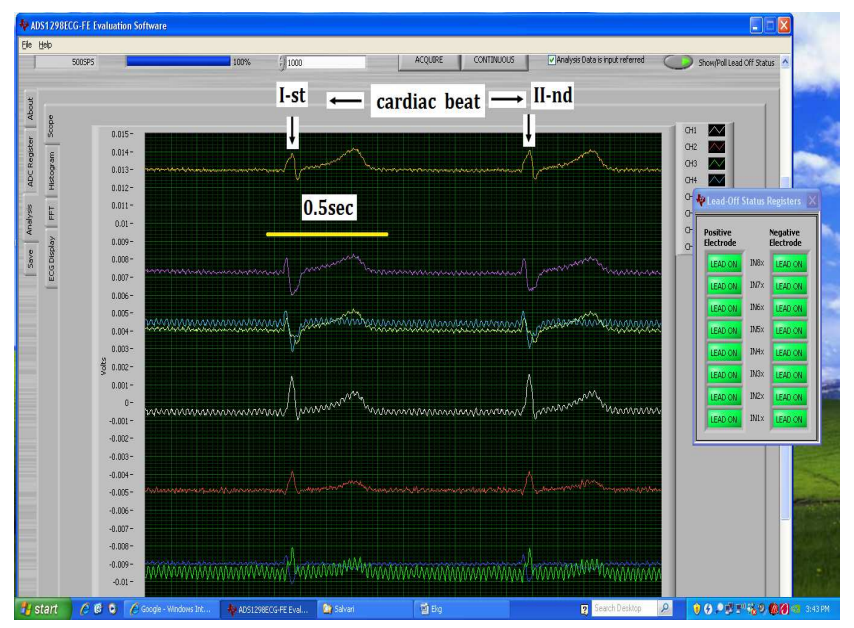

Figure 14. The cropped electrophysiological signals, using the Display mode for ADS1298.

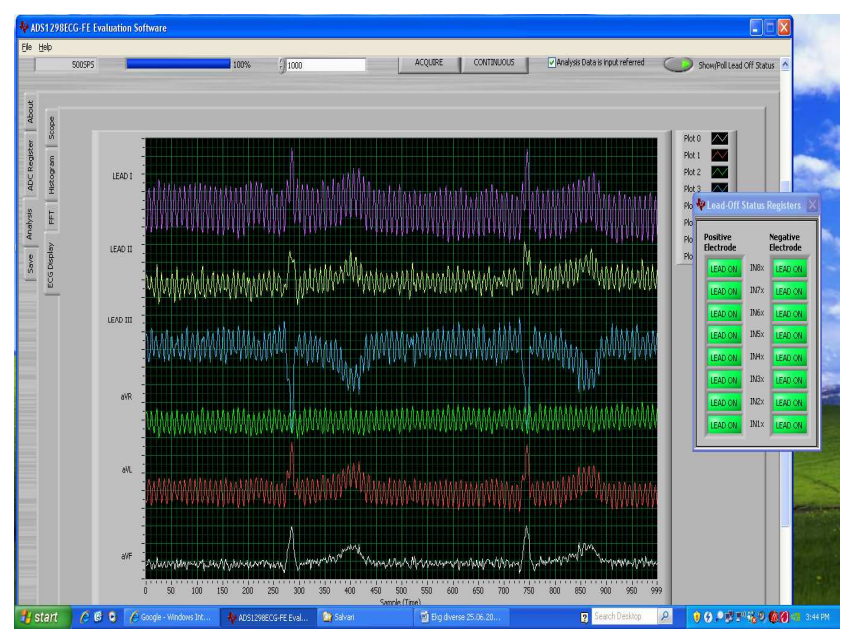

Figure 15. GPIO register inside its window. The cropped electrophysiological signals, using the Scope mode for ADS1298, selecting amplification $=1$.

In next picture, the cropped signals are processed using Fast Fourier Transform FFT, fig. 16. Investigating the frequency spectrum, can be discovered those important spectrum components, like ECG trace corresponding to $\sim 1 \mathrm{~Hz}$ and EMG corresponding to $\sim 35 \mathrm{~Hz}$. Figure 17 presents the histogram analysis performed by ADS1298ECGFE-PDK Version 1.0.0 software. In this way, the main spectral components are obviously and can be further amplified. This analysis becomes very useful for next improvements using digital filters that have to amplify only few desired targeted harmonics.

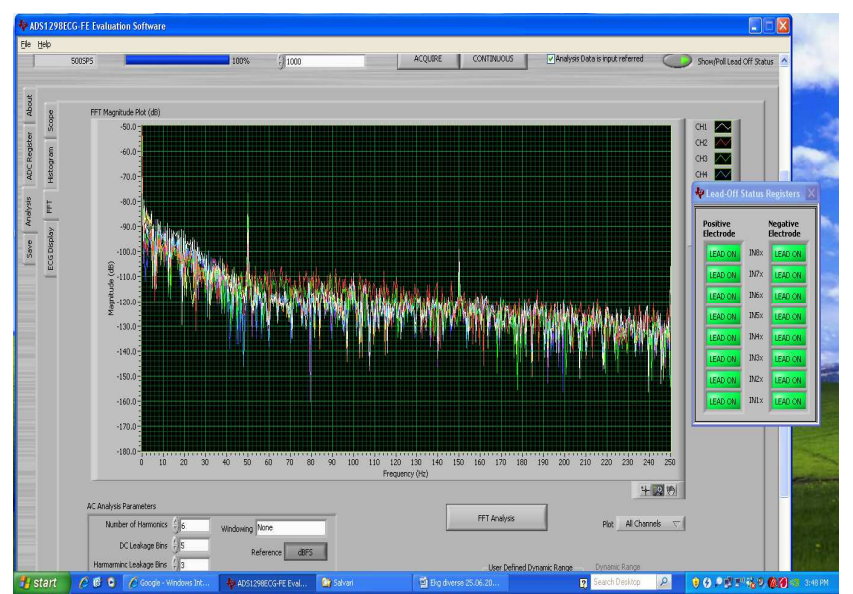

Figure 16. The FFT analysis of the cropped electrophysiological signal from abdominal skin.

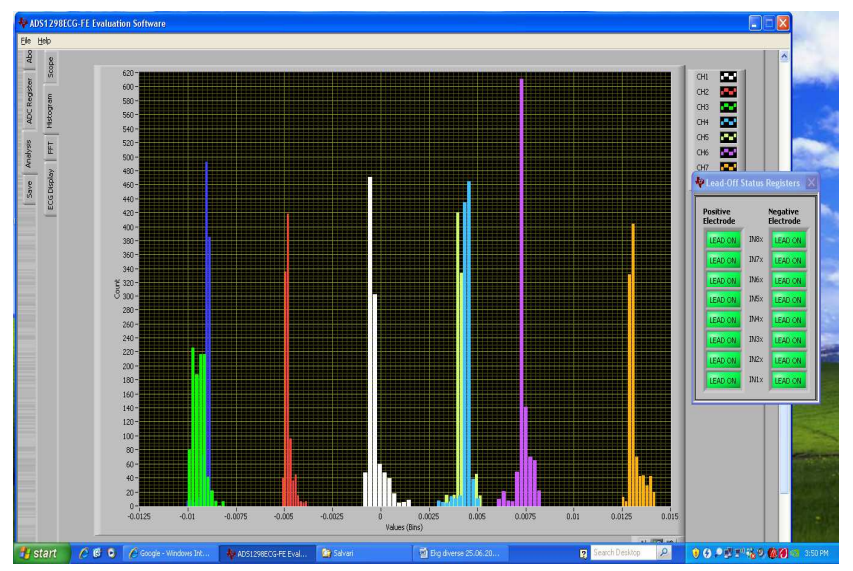

Figure 17. Histogram of the previous cropped signal.

The FFT analysis probes that our electrophysiological equipment is sensitive also to the network $50 \mathrm{~Hz}$ frequency and to the cardiac rhythm. The proof of the ECG signal detection consists in a horizontal distance of 450 samples. Taking into account that the sample rate is 500SPS, results a cardiac frequency equal with 1.101 beats/second or 67 beats/minute.

\section{Conclusions}

This paper approached interferences of general electrophysiological techniques with applications in public health monitoring regarding the intern organs measuring and characterisation like ECG, EMG, EEG, neural signals and psychiatry. The original part of the paper was focused on new hardware and software solution development. The biosignal processing involves a clipper and a selective frequencies amplifier, because the electrophysiological signals usually have a high dynamic range and can be easily covered by the artefacts noise.

The presented vector data collection, processing and recording had the possibility to use many input channels that give the possibility to simultaneously test different versions of source-electrodes-amplifier blocks. Later this facility can be used to multipoint measuring or to increase the resolution. A specific data vector recording was presented, with the 
advantage of development for future new digital processing.

\section{Acknowledgements}

The work was co-funded by PN2 contract no. 35/2014 EL081401 MEDICY.

\section{References}

[1] Květina, J.; Varayil, J.E; Ali, S.M; Kuneš, M.; Bureš, J.; Tachecí, I.; Rejchrt, S. \& Kopáčová, M., Preclinical electrogastrography in experimental pigs. International Journal of Interdiscip. Toxicol., Vol.3, No.2, (June 2010), pp. 53-58.

[2] C. Costa Santos, J. Bernardes, P.M.B. Vitanyi, L. Antunes. Clustering fetal heart rate tracing by compression. 2006, pp.1-6, available at

[3] http://arxiv.org/ftp/q-bio/papers/0612/0612013.pdf

[4] Bogdan, D.; Craciun, M.; Dochia, R.I.; Ionescu, M.A. \& Ravariu, C. (2009). Circuit design for noise rejection in electromyography, Proc. of INGIMED 2009 2nd National Conference on Biomedical Engineering, pp. 76-81, Bucharest, Romania, ICPE-CA Publisher, November 12-14, 2009

[5] D. Careglio, J. Solé Pareta, S. Spadaro. Service category-to-wavelength selection technique for QoS support in connection-oriented optical packet switching, The International Journal of Computer and Telecommunications Networking, Volume 51 Issue 1, 17 January 2007. DOI: 10.1016/j.comnet.2006.04.008.

[6] B. Firtat, R. Iosub, D. Necula, F. Babarada, E. Franti, C. Moldovan, (2008) Simulation, design and microfabrication of multichannel microprobe for bioelectrical signals recording, IEEE Int. Conf., Sinaia, Romania, 2008, pp 177-180.

[7] F. Babarada, J. Arhip, (2009) Electrophysiology Signal Data Vector Acquiring, Congress of Romanian Medical Association, Bucharest, 2009, pp . 82-83

[8] Fen-Ying Kong, Sai-Xi Gu, Wei-Wei Li, Ting-Ting Chen, Qin $\mathrm{Xu}$, Wei Wang, A paper disk equipped with graphene/polyaniline/Au nanoparticles/glucose oxidase biocomposite modified screen-printed electrode: Toward hole blood glucose determination, Biosensors and Bioelectronics, Volume 56, 15 June 2014, Pages 77-82.

[9] Ravariu, R. Botan, The Electrical Transport Mechanisms Investigation In Adrenergic Synapses Using A Parallel BioOI Biodevice, 19th IEEE International EURASIP Conference of Biosignal, Brno, Czech Republic, under IEEE Engineering in Medicine and Biology Society, Jun. 29-30. 2008, pp.63.115-118.

[10] Ricardo M. Santos, João Laranjinha, Rui M. Barbosa, Anton Sirota, Simultaneous measurement of cholinergic tone and neuronal network dynamics in vivo in the rat brain using a novel choline oxidase based electrochemical biosensor, Biosensor and Bioelectronics, vol. 69, no. 7, July 2015, pp. 83-94.

[11] Mohamed Basiouny Alghannam, Mohamed Ramadan El-Kholy, Ashraf Anas Zytoon, Ayman Mohamed Ebied, The Role of MRI and MR Arthrography in Evaluation of Wrist Pain, International Science PG Journal of Medical Imaging. Vol. 2, No. 6, 2014, pp. 149-155.
[12] C. Ravariu, D. Ursutiu, F. Babarada, J. Arhip, S. S. Arama, G. Radulian, C. Samoila. Remote Measurements of the Gastric Electrical Signals - Between Theory and Practice, 11-th Int. IEEE Conference on Remote Engineering and Virtual Instrumentation, Porto, Portugal, Proceedings pp. 281-284, 2014.

[13] Ahmed Saad Al-Ghabban, Abbas Fathil Al-Hashimi, Ziad Tariq Al-Dahan, Predominant Peak Detection of QRS Complexes, International Science PG Journal of Medical Imaging. Vol. 2, No. 6, 2014, pp. 133-137.

[14] Wenqiong Xue, Jian Kang, F. DuBois Bowman, Tor D. Wager, and Jian Guo, Identifying functional co-activation patterns in neuroimaging studies via Poisson graphical models, Biometrics, Vol. 70, No. 4, pp. 234-239, December 2014.

[15] Cristian Ravariu, Ala Bondarciuc. The sensitivity in the IR spectrum of the intact and pathological tissues by laser bio-photometry, Laser in Medical Science, Springer Journal, March 2014, Volume 29, Issue 2, pp 581-588.

[16] Shah S, Bougherara H, Schemitsch EH, Zdero R (2012) Biomechanical stress maps of an artificial femur obtained using a new infrared thermography technique validated by strain gages. Medical 553 Engineering \& Physics, 2012, vol. 34, pp. 1496-1502.

[17] Rowena Jacob, Kiriakos Xenitidis and Colin Campbell, How to interpret different results for CRHTT data, The British Journal of Psychiatry, (2011) 199, 249-254.

[18] M. G.-Serbanescu, C. Diaconu, S. Herms, C. Bleotu, J. Vollmer, T.W. Muhleisen, D. Prelipceanu,L. Priebe, R. Mihailescu, M-J. Georgescu, D. Sima, M. Grimberg, M. M. Nothen and S. Cichon, Investigation of the tryptophan hydroxylase 2 gene in bipolar I disorder in the Romanian population, Psychiatric Genetics, Oct. 2008, vol. 18, no.5, pp. 240-247.

[19] Mohammad Movassat, Nasser Ashgriz, Bob Cheung, Blast Wave Induced Flows in Semicircular Canals, Science PG American Journal of Bioscience and Bioengineering. Vol. 3, No. 1, 2015, pp. 1-7.

[20] C. Ravariu, C.I. Tirgoviste, O. Dumitrache. The modeling of the insulin exocytosys after a glycemic stimulus, paper ID 723-088, 8-th IASTED International Conference Biomedical Engineering BioMED, 2011, Innsbruck, Austria, pp. 188-191.

[21] C. Ravariu, A. Bondarciuc, V. Nahaba, V. Bondarciuc, C. I. Tirgoviste, F. Babarada, O. Dumitrache. The laser biophotometry medical applications for the diabetes foot monitoring, Elsevier Journal of Photodyagnosis and Photodynamic Therapy, vol. 7, suppl. 1, pp. 522-523, July 2010.

[22] Michael Davidson, Silvana Galderisi, Mark Weiser, Nomi Werbeloff, Wolfgang W. Fleischhacker, Richard S. Keefe, Han Boter, Ireneus P.M. Keet, Dan Prelipceanu, Janusz K. Rybakowski, Jan Libiger, Martina Hummer, Sonia Dollfus, Juan J. López-Ibor, Luchezar G. Hranov, Wolfgang Gaebel, Joseph Peuskens, Nils Lindefors, Anita Riecher-Rössler, René S. Kahn, Cognitive Effects of Antipsychotic Drugs in First-Episode Schizophrenia and Schizophreniform Disorder: A Randomized, Open-Label Clinical Trial EUFEST, American Journal of Psychiatry, June 2009 Vol. 166, no. 6, pp. 675-682.

[23] C. Ravariu, C. Ionescu-Tirgoviste, F. Ravariu. Glucose biofuels properties in the bloodstream in conjunction with the beta cell electro-physiology, Proceedings of 2-nd Edition IEEE - ICCEP International Conference on Clean Electrical Power Conference, Jun. 09-11. 2009, Capri, Italy, pp. 124-127. 
[24] Armida Mucci, Umberto Volpe, Nash Boutros, Evidence-Based Medicine and Electrophysiology in Schizophrenia, Clinical EEG and Neuroscience, April 2009 vol. 40, no. 2, pp. 62-77.

[25] Gerard J. Marek, George K. Aghajanian, The electrophysiology of prefrontal serotonin systems: therapeutic implications for mood and psychosis, Biological Psychiatry, Dec 1998, vol. 44, no 11, pp. 1118-1127.

[26] Coles, Michael G.H.; Michael D. Rugg (1996). "Event-related brain potentials: an introduction". Electrophysiology of Mind. Oxford Scholarship Online Monographs. pp. 1-27.

[27] Carol Jahshan1,2, Jonathan K. Wynn1,2, Kristopher I. Mathis1,2 \& Michael F. Green, The neurophysiology of biological motion perception in schizophrenia, Brain and Behavior, doi: 10.1002/brb3.303, pp. 76 - 84.

[28] Robert J Barry, Adam R Clarke, Stuart J Johnston, A review of electrophysiology in attention-deficit/hyperactivity disorder: I. Qualitative and quantitative electroencephalography, Clinical Neurophysiology, Volume 114, Issue 2, February 2003, Pages 171-183.
[29] Lenartowicz A, Delorme A, Walshaw PD, Cho AL, Bilder RM, McGough JJ, McCracken JT, Makeig S, Loo SK, Electroencephalography correlates of spatial working memory deficits in attention-deficit/hyperactivity disorder: vigilance, encoding, and maintenance, Journal of Neuroscience, Jan 2014 22; 34(4), pp. 1171-82.

[30] Rustem Popa, (2006) Medical Electronics. Matrix House, Bucharest Publishing House, Romania, in Romanian.

[31] ATMega32 data sheet, available at $\mathrm{http}: / /$ www.atmel.com/dyn/resources/prod documents/doc2503.pdf

[32] T-Y. Tu, C-Y. Chen, D-S. Jong and A. M. Wo, "An integrated electrophysiological and optical approach for ion channel study in a microfluidic system enabling intra- and extra-cellular solution exchange", Sensors and Actuators B: Chemical, vol. 185, pp. 496-503, August 2013. 